\title{
Percepción de pacientes con VIH/Sida sobre consulta odontológica en IPS de Santa Marta - Colombia
}

\author{
Perception of patients with HIV / AIDS on dental consultation in Santa Marta - Colombia \\ Luis Armando Vila-Sierra' ${ }^{1}$ orcid.org/0000-0003-3888-1061 \\ María Teresa Hernandez-Fuentes ${ }^{*}$ orcid.org/0000-0003-4868-2031
}

1 Facultad de Ciencias de Salud, Universidad del Magdalena. Santa Marta, Colombia

Fecha de recepción: Abril 10 - 2018

Fecha de revisión: Julio 4 - 2019

Fecha de aceptación: Abril 30 - 2020

Vila-Sierra LA, Hernández-Fuentes MT. Percepción de pacientes con VIH/Sida sobre consulta odontológica en IPS de Santa Marta - Colombia. Univ. Salud. 2020;22(2):120-126. DOI: https://doi.org/10.22267/rus.202202.183

\section{Resumen}

Introducción: La atención odontológica oportuna e inclusiva tiene un papel importante en las personas con VIH/SIDA, por cuanto permite prevenir y dar tratamiento a las múltiples lesiones orales que acompañan a esta patología, mejorando la calidad de vida de esta población. Objetivo: Determinar la percepción y experiencia de pacientes con VIH/SIDA sobre la consulta odontológica en una Institución Prestadora de Salud (IPS) de Santa Marta, Colombia. Materiales y métodos: Se realizó un estudio descriptivo cuantitativo donde participaron 64 pacientes con VIH/SIDA de una IPS de la ciudad de Santa Marta, quienes se les aplicó un instrumento de recolección de datos. Resultados: El 65,6\% expresó nunca percibir rechazo por parte del odontólogo, el 25\% refiere haber experimentado algún rechazo por lo menos una vez y un 9,4\% siempre se siente rechazado. Conclusiones: Existe la necesidad de abordar esta enfermedad no sólo desde el aspecto clínico, sino también desde lo social, para educar tanto al profesional de la salud como a la comunidad, sobre avances científicos, el estudio de esta patología y los riesgos reales de contraerla, para desmitificar esta afección y erradicar la discriminación hacia los pacientes.

Palabras clave: VIH; Síndrome de Inmunodeficiencia Adquirida (SIDA); percepción; atención odontológica. (Fuente: DeCS, Bireme).

\begin{abstract}
Introduction: Timely and comprehensive dental care is important for HIV/AIDS patients as it facilitates prevention and treatment of the multiple oral lesions that accompany this pathology, and consequently, improves their quality of life. Objective: To determine the perception and experience of the dental care service provided by a Health Provider Institution (HPI) to HIV/AIDS patients from the city of Santa Marta (Colombia). Materials and methods: A descriptive quantitative study was carried out with 64 HIV/AIDS patients treated in the HPI, to whom a data collection instrument was applied. Results: $65.6 \%$ of the HIV/AIDS patients did not perceive any rejection from the dentist, whereas $25 \%$ and $9.4 \%$ of these patients reported being discriminated at least once and always, respectively. Conclusions: There is a need to approach this disease not only from a clinical standpoint but also from a social perspective in order to educate both health professionals and communities about: scientific advances; how to study this pathology; and the risks of contracting HIV/AIDS. It is also necessary to demystify some misconceptions and eradicate discrimination against these patients.
\end{abstract}

Key words: HIV; Acquired Immunodeficiency Syndrome (AIDS); perception; dental care. (Source: DeCS, Bireme).

\footnotetext{
*Autor de correspondencia

María Teresa Hernández Fuentes

e-mail: mariateresa-hernandez@hotmail.com
} 


\section{Introducción}

El virus de la inmunodeficiencia humana (VIH) afecta a las células encargadas de la defensa del organismo, ocasionando que estas no cumplan con la función de mantener protegido al individuo, llevándolo a un estado de insuficiencia inmunitaria y que múltiples patologías deterioren su salud. Entre las manifestaciones clínicas se encuentran las lesiones orales que pueden estar presentes hasta en el $50 \%$ de las personas con infección por VIH y en 80\% de aquellas con diagnóstico de Síndrome de Inmunodeficiencia Adquirida (SIDA)(1). Esto justifica la necesidad ofrecer servicios de salud de calidad, que garanticen el uso adecuado de medidas de bioseguridad para proteger al paciente $\mathrm{y}$ al profesional de salud(2,3).

En Colombia, tras la aplicación de las guías clínicas para la prevención, diagnóstico y tratamiento de pacientes con VIH/SIDA, referidas en la Resolución 3442 de 2006 se ha reportado una disminución en los casos de contagio en trabajadores de la salud (1\%) por heridas percutáneas con objetos corto punzantes $(4,5)$.

El reporte del programa conjunto de las Naciones Unidas sobre el VIH/SIDA (ONUSIDA) informó que en 2018 se produjeron 1,7 millones de nuevas infecciones por VIH, cifra menor a la registrada en 1997 (2,9 millones); asimismo la mortalidad por el SIDA ha disminuido un 33\% desde 2010(6). Pese a esto, aún existe una percepción social negativa hacia las personas con VIH/SIDA, que genera un rechazo hacia esta comunidad $(7,8)$.

En Colombia el Decreto 1543 de 1997, establece las actividades diagnósticas, de promoción y prevención, control epidemiológico de la infección por el VIH, el SIDA y otras Enfermedades de Transmisión Sexual (ETS)(9). La prestación de servicios de salud en todos los niveles de complejidad a pacientes convivientes con VIH/SIDA, es de carácter obligatorio y debe garantizar el uso de medidas de bioseguridad que protejan al paciente como al profesional que presta el servicio(10).

Por otra parte, el odontólogo es considerado como personal de alto riesgo a contraer infecciones, debido al uso de múltiples instrumentos corto punzantes, la exposición a microorganismos, sangre contaminada y secreciones bucales ${ }^{(11)}$.
Probablemente esto incrementa la percepción de peligro del odontólogo, dado que es una enfermedad mortal y se puede contraer durante el desarrollo de la actividad laboral(12). En relación a esto, algunos estudios sustentan que entre el $15 \%$ y $35 \%$ de odontólogos y/o estudiantes de odontología muestran una postura negativa frente a pacientes $\mathrm{VIH}+(13,14)$.

Por tanto, y dado que la discriminación y el prejuicio afecta emocionalmente al individuo y disminuye su calidad de vida(15), es necesario resaltar la importancia que representa para estos pacientes contar con un servicio odontológico oportuno con condiciones igualitarias $(16,17)$.

El objetivo de este estudio fue determinar cuál es la percepción y experiencia de los pacientes con VIH/SIDA sobre la consulta odontológica en una IPS de la ciudad de Santa Marta, de esta manera obtener información que permita brindar elementos para la toma de decisiones en temas de prestación oportuna de servicios que mejoren su calidad de vida.

\section{Materiales y métodos}

\section{Tipo de estudio y población}

Se realizó una investigación de tipo descriptiva cuantitativa que comprende la presentación, registro y análisis de la naturaleza actual del problema ${ }^{(18)}$.

La población estuvo conformada por 64 pacientes diagnosticados con VIH/SIDA, pertenecientes a una Institución Prestadora de Salud (IPS) de la ciudad de Santa Marta, Colombia, quienes cumplieron los siguientes criterios de inclusión: Ser mayor de 18 años sin predilección de género, estar diagnosticado con VIH/SIDA, haber asistido a la consulta odontológica en los últimos 12 meses previos al proyecto de estudio y aceptar de forma voluntaria su participación. Los criterios de exclusión fueron: no cumplir con los requisitos anteriores o ser paciente en fase terminal de la enfermedad.

\section{Proceso de medición y recolección}

Se elaboró una encuesta que fue validada por parte de tres expertos, entre ellos un doctor en psicobiología, una especialista en neurociencia y biología del comportamiento, y especialista en cirugía oral y estomatología. El instrumento fue conformado por tres secciones, la primera con información sociodemográfica de los pacientes, la 
segunda exploraba la variable percepción y la tercera, la variable experiencia en relación a la frecuencia de los sucesos, así: nunca, casi nunca, a veces, casi siempre y siempre.

\section{Análisis estadístico}

Los datos obtenidos se digitaron en el programa Microsoft Excel® y el análisis se realizó con el paquete estadístico SPSS versión 20, utilizando como medidas estadísticas promedio, desviación estándar, porcentajes, y el test de $C h{ }^{2}$ para determinar la significancia estadística que fue de 0,05.

\section{Consideraciones éticas}

Esta investigación contó con la aprobación del representante legal de la IPS donde se llevó a cabo el estudio, el aval del Comité de Ética en Investigación de la Universidad del Magdalena, quien conceptuó que la propuesta de investigación cumplía con los aspectos éticos y se catalogó "sin riesgo", según lo establecido en la Resolución 8430 de 1993(19) de la normatividad colombiana y la declaración de Helsinki. Se explicó a los pacientes el objeto de la investigación, quienes firmaron el consentimiento informado, el cual contempla la confidencialidad y el anonimato de cada participante del estudio.

\section{Resultados}

Las características sociodemográficas de los participantes fueron: El 37,5\% de los individuos se encontraba entre el rango de 18 a 32 años, el 34,4\% de 33-47 años, el 28,1\% de 48 a 62 años, con un predominio del sexo masculino en un $51 \%$. El $65,6 \%$ de los pacientes expresaron nunca haber sentido rechazo en la consulta odontológica (Tabla 1).

Se encontró que el principal motivo de consulta odontológica fue limpieza oral (34\%), seguido por el dolor dental (26\%), rehabilitación oral (25\%), gingivorragia (6\%), cita de control (9\%). Acerca de informar al odontólogo sobre su condición serológica, se encontró que el 58\% manifestó que siempre refería ser paciente conviviente con VIH, el 23\% expresó que nunca informa al odontólogo, mientras un $19 \%$ lo hace de manera ocasional.

En lo que concierne a la actitud del odontólogo durante la consulta, el $67,2 \%$ de las personas percibió una conducta amable, contrastando al 1,6\% quien refiere que éste fue agresivo (Tabla 2).
El 62,4\% de los integrantes del estudio informó que al notificar serodiagnóstico positivo para VIH, siempre reciben atención odontológica, contrario al $11 \%$ que afirmó que le han negado la consulta y el $26,6 \%$ expresó que por lo menos una vez le han privado de recibir el servicio odontológico (Tabla 3).

Tabla 1. Percepción de rechazo por parte del odontólogo durante la atención

\begin{tabular}{lrr}
\hline \multicolumn{1}{c}{ Ítems } & Frecuencia & $\%$ \\
\hline Nunca & 42 & 65,6 \\
Casi nunca & 5 & 7,8 \\
A veces & 10 & 15,6 \\
Casi siempre & 1 & 1,6 \\
Siempre & 6 & 9,4 \\
Total & 64 & 100 \\
\hline
\end{tabular}

Tabla 2. Actitud del odontólogo durante la consulta

\begin{tabular}{lrr}
\hline \multicolumn{1}{c}{ Ítems } & Frecuencia & \% \\
\hline Amable & 43 & 67,2 \\
Impaciente & 8 & 12,5 \\
Indiferente & 7 & 10,9 \\
Tranquilo & 5 & 7,8 \\
Agresivo & 1 & 1,6 \\
Total & 64 & 100 \\
\hline
\end{tabular}

Ante estos resultados el $58 \%$ de los participantes expresó estar de acuerdo con la implementación de consultas odontológicas especiales para pacientes que viven con VIH/SIDA en otras instituciones de salud. Un $16 \%$ afirmó que es conveniente brindar ocasionalmente este tipo de consultas focalizadas, sin embargo, para el $26 \%$ no resulta una estrategia incluyente. Asimismo, el 25\% de los pacientes entre 48 a 62 años afirma siempre ser atendidos, aun cuando se identifiquen como VIH+ (Tabla 3 ).

En la Tabla 4 se puede apreciar la asociación entre edad y el considerar que la consulta odontológica fuera diferente debido a su condición de VIH; al respecto más del $20 \%$ de los pacientes entre 33 a 47 años nunca ha considerado que la consulta odontológica sea distinta a causa de su patología. 
Tabla 3. Edad - Lo atienden en la consulta odontológica aunque se identifique como VIH+

\begin{tabular}{|c|c|c|c|c|c|c|c|c|c|}
\hline Edad & $\begin{array}{c}\% \\
\text { Nunca }\end{array}$ & $\begin{array}{l}\text { \% Casi } \\
\text { Nunca }\end{array}$ & $\%$ A veces & $\begin{array}{l}\% \text { Casi } \\
\text { siempre }\end{array}$ & $\begin{array}{c}\% \\
\text { Siempre }\end{array}$ & Total & Valor & Gl & $\begin{array}{l}\text { Sig. asintótica } \\
\text { (bilateral) }\end{array}$ \\
\hline De 18 a 32 años & 4,7 & & 7,8 & 7,8 & 17,2 & 37,5 & & & \\
\hline De 33 a 47 años & 6,3 & 1,6 & 6,3 & & 20,2 & 34,4 & & & \\
\hline De 48 a 62 años & & & 3,1 & & 25 & 28,1 & & & \\
\hline Total & 11 & 1,6 & 17,2 & 7,8 & 62,4 & 100 & & & \\
\hline \multicolumn{10}{|l|}{ Pruebas de $\mathrm{Chi}^{2}$} \\
\hline Chi ${ }^{2}$ de Pearson & & & & & & & $17,018^{a}$ & 8 & 0,030 \\
\hline Razón de verosimilitudes & & & & & & & 20,429 & 8 & 0,009 \\
\hline $\mathrm{N}$ de casos válidos & & & & & & & 64 & & \\
\hline
\end{tabular}

Tabla 4. Edad - Consideró que la consulta fue diferente debido a su condición de VIH+

\begin{tabular}{|c|c|c|c|c|c|c|c|c|c|}
\hline Edad & $\begin{array}{c}\% \\
\text { Nunca }\end{array}$ & $\begin{array}{l}\text { \% Casi } \\
\text { nunca }\end{array}$ & $\%$ A veces & $\begin{array}{c}\% \text { Casi } \\
\text { siempre }\end{array}$ & $\begin{array}{c}\% \\
\text { Siempre }\end{array}$ & Total & Valor & Gl & $\begin{array}{l}\text { Sig. asintótica } \\
\text { (bilateral) }\end{array}$ \\
\hline De 18 a 32 años & 17,2 & 1,6 & 12,5 & 3,1 & 3,1 & 37,5 & & & \\
\hline De 33 a 47 años & 25 & & 4,7 & 1,6 & 3,1 & 34,4 & & & \\
\hline De 48 a 62 años & 20,2 & 1,6 & 1,6 & 0 & 4,7 & 28,1 & & & \\
\hline Total & 62,4 & 3,2 & 18,8 & 4,7 & 10,9 & 100 & & & \\
\hline \multicolumn{10}{|l|}{ Pruebas de $C h i^{2}$} \\
\hline $\mathrm{Chi}^{2}$ de Pearson & & & & & & & $9,801^{a}$ & 8 & 0,279 \\
\hline Razón de verosimilitudes & & & & & & & 11,313 & 8 & 0,185 \\
\hline $\mathrm{N}$ de casos válidos & & & & & & & 64 & & \\
\hline
\end{tabular}

\section{Discusión}

La salud oral tiene un papel fundamental en las personas que viven con VIH/SIDA, teniendo en cuenta que los pacientes con VIH y aproximadamente el $90 \%$ de pacientes con SIDA, tienen alguna patología bucal asociada(20). Por ello, es necesario la atención del odontólogo, no solo frente a las lesiones orales, sino también en promoción y prevención para la salud oral de esta población(21,22).

Dado que en los últimos años el VIH/SIDA se presenta entre personas de diferentes edades, sexo, niveles socioeconómicos, se incrementa la atención odontológica(23), como lo refieren Ramírez, et al.(24), quienes encontraron que el motivo de consulta más frecuente son los problemas dentarios $46,6 \%$ y la proporción de pacientes que refieren ser VIH+ es del 64,3\%; datos similares a los reportados por el presente estudio, donde la limpieza fue el motivo de consulta más habitual (34\%) y la notificación de la condición VIH+ fue del 58\%. Sin embargo, el 23\% de la población comentó que nunca lo hace, posiblemente por la percepción de rechazo hacia su condición serológica.

García, et al.(25), mencionan en su estudio que estas personas presentan dificultades para acceder a la consulta odontológica, que pueden estar asociadas a la discriminación por parte del odontólogo y a una atención inadecuada, coincidente con lo planteado por Cesar, et al.(26), quienes encontraron que aún existen prejuicios e ignorancia sobre el riesgo de infección por VIH/SIDA. De la misma manera, Dávila, et al.(27), evidenciaron las experiencias negativas de pacientes en relación a la consulta odontológica ${ }^{(28,29)}$.

No obstante, el presente estudio, reportó que el $65,6 \%$ de los participantes refirieron nunca haber sentido rechazo en la consulta, esto por cuanto son adscritos a instituciones prestadoras de servicios de 
salud (IPS) específicas, que les brindan los servicios médicos y odontológicos, lo cual mejora las condiciones de la atención.

Tamayo-Zuluaga, et al.(30), afirman en su estudio que el estigma es mayor en las personas con menor edad y que el $50 \%$ de valores centrales osciló entre $19 \mathrm{y}$ 24 años, y el rango entre 16 a 59 años, así mismo este estudio revela que la percepción de rechazo es más alta en los rangos etarios de 18 a 32 y 33 a 47 años con un $4,7 \%$ y $6,3 \%$ respectivamente, quienes afirman que les han negado la atención por ser pacientes convivientes con VIH.

Según Dávila, et al.(13), la actitud a la prestación de servicios odontológicos a personas que viven con VIH/SIDA de algunos profesionales de la salud, específicamente no mostró diferencias entre el número de odontólogos que presentaban una postura favorable hacia los pacientes $(41,7 \%)$ y una actitud desfavorable con el 43,8\%. Sin embargo, este estudio reveló que un $67,2 \%$ de los participantes, expresaron que la actitud del odontólogo fue amable, de lo cual se puede concluir que en términos generales la conducta del odontólogo es confortable hacia el paciente, pero aún sigue persistiendo en menor escala rechazo al momento de ofrecer la atención.

Estudios previos como el de Elizondo JE, et al. (31) en su investigación, La odontología y el estigma asociado al VIH han concluido que existe un alto nivel de aceptación para trabajar con este grupo de pacientes siendo poco el rechazo, dándole así soporte al resultado de este estudio, en el que se evidenció que el grado de rechazo es bajo y la percepción durante la consulta odontológica es favorable.

\section{Conclusión}

A pesar de que la percepción y experiencia de pacientes con VIH/SIDA que acuden a la consulta odontológica es buena, aun es necesario abordar esta patología desde otra perspectiva, de manera que se confronte mitos acerca del contagio y eduque a la población en general.

Por otra parte, se recomienda continuar con investigaciones sobre la percepción y experiencia del profesional de salud.

\section{Conflicto de interés}

Los autores no reportan conflicto de interés, como tampoco ninguna fuente de financiamiento para la realización de la investigación.

\section{Referencias}

1. Donoso-Hofer F. Lesiones orales asociadas con la enfermedad del virus de inmunodeficiencia humana en pacientes adultos, una perspectiva clínica. Rev. chil. infectol [Internet]. 2016 Oct [citado 2019 Nov 20];33( Suppl 1):27-35. Disponible en: https://scielo.conicyt.cl/scielo.php?script=sci_arttext\&pid= S0716-10182016000700004\&lng=es

2. Organización STOPVIH. Cuidados odontológicos en personas con VIH. Informe de un grupo de científicos de STOPVIH. Venezuela: STOPVIH; 2013. Recuperado a partir de: http://www.stopvih.org/noticias/cuidados-odontologicosen-personas-con-vih/

3. Ministerio de Salud y Protección Social. Guía de práctica clínica (GPC) basada en la evidencia científica para la atención de la infección por VIH/Sida en adolescentes (con 13 años o más de edad) y adultos. Bogotá: MinSalud; 2014. Recuperado a partir de: https://www.ins.gov.co/buscadoreventos/Informesdeevento/VIH-SIDA\%202017.pdf

4. Esquivel L, Fernández L, Magis C. Actitudes de rechazo hacia el paciente infectado por el VIH, en la práctica odontológica. Rev. ADM [Internet]. 2006 [citado 2015 Oct 20];57(6):214217. Recuperado a partir de: http://www.medigraphic.com/pdfs/adm/od2000/od006c.pdf

5. Ministerio de Salud y Protección Social. Resolución N ${ }^{\circ} 3442$ de 2006 Por la cual se adoptan las Guías de Práctica Clínica basadas en evidencia para la prevención, diagnóstico y tratamiento de pacientes con VIH / SIDA y Enfermedad Renal Crónica y las recomendaciones de los Modelos de Gestión Programática en VIH/SIDA y de Prevención y Control de la Enfermedad Renal Crónica [Internet]. Bogotá: MinSalud; 2006. Recuperado a partir de: https://www.minsalud.gov.co/Normatividad_Nuevo/RESOL Uci\%C3\%93N\%203442\%20DE\%202006.pdf

6. ONUSIDA. Programa conjunto de las naciones unidas sobre el VIH/SIDA. Hoja informativa-actualización global sobre el Sida 2019. Informe de un grupo de científicos de la ONUSIDA. Suiza: ONUSIDA; 2019. Recuperado a partir de: https://www.unaids.org/sites/default/files/media_asset/U NAIDS_FactSheet_es.pdf

7. Cabré S, Mujica M, Dávila M. Experiencias estigmatizantes de las personas que viven con VIH/SIDA en el contexto de la consulta odontológica. Educere [Internet]. 2012 [citado 2015 Oct 20];16(55):355-360. Recuperado a partir de: http://www.redalyc.org/articulo.oa?id=35626140018> ISSN 1316-4910

8. Betancourt García A, Nodal García C, García Rodríguez J, Ferrer Vilches D, Álvarez Heredia E. Ética y bioseguridad en la atención estomatológica al paciente con virus de inmunodeficiencia humana. Medisur [Internet]. 2013 Feb [citado 2018 Abr 05];11(1):44-53. Recuperado a partir de: http://scielo.sld.cu/scielo.php?script=sci_arttext\&pid=S172 7-897X2013000100007\&lng=es 
9. Ministerio de Salud y Protección Social. Decreto número 1543 de 1997 Por el cual se reglamenta el manejo de la infección por el Virus de Inmunodeficiencia Humana (VIH), el Síndrome de la Inmunodeficiencia Adquirida (SIDA) y las otras Enfermedades de Transmisión Sexual (ETS) [Internet]. Bogotá: MinSalud; 1997. Recuperado a partir de: https://www.minsalud.gov.co/sites/rid/Lists/BibliotecaDig ital/RIDE/DE/DIJ/Decreto-1543-de-1997.pdf

10. Acosta B. Atención al paciente VIH/SIDA: legislación y bioseguridad odontológica en Colombia. Acta bioeth [Internet]. 2006 [citado 2015 Oct 20];12(1):23-28. Recuperado a partir de: http://dx.doi.org/10.4067/S1726569X2006000100003

11. Tovar V, Guerra ME, Carvajal A. Accidentes laborales y riesgo a contraer infección por el Virus de Inmunodeficiencia humana y el Virus de la Hepatitis B y C en el consultorio Odontológico. Acta odontol. venez [Internet]. 2004 Sep [citado 2019 Nov 21];42(3):218-225. Disponible en: http://ve.scielo.org/scielo.php?script=sci_arttext\&pid=S000 1-63652004000300013\&lng=es

12. Acosta de Velásquez Blanca Lucía, Pérez-Then Eddy, Mueses Marín Héctor Fabio, Pinzón Gómez Elisa María, Shor-Posner Gail. Actitud de los estudiantes en tres facultades de odontología en Santiago de Cali, Colombia, frente a pacientes con VIH. Acta bioeth. [Internet]. 2011 Nov [citado 2019 Nov 20];17(2):265-271. Disponible en: http://dx.doi.org/10.4067/S1726-569X2011000200013

13. Dávila M, Gil M. Nivel de conocimiento y actitud de los odontólogos hacia portadores de VIH/SIDA. Acta odontol. Venez [Internet]. 2007 [citado el 2015 Oct 20];45(2):1-9. Recuperado a partir de: http://www.actaodontologica.com/ediciones/2007/2/nivel _conocimiento_actitud.asp

14. Conde Higuera P, Pimentel Ramírez ML, Díaz Ávila A, Domingo Moratalla T. Stigma, discrimination and adherence to treatment of children with HIV and AIDS: A bioethical perspective. Acta bioeth. [Internet]. 2016 Nov [citado 2018 Abr 05];22(2):331-340. Recuperado a partir de: http://dx.doi.org/10.4067/S1726-569X2016000200020

15. Marín M, Garrido M. La interacción comunicativa en los grupos. Madrid: Pirámide; 2003

16. Sans Cueto $\mathrm{Y}$, Martínez Rodríguez M, Blanco Hernández 0 , Labrador Falero DM, Acosta Lopez Y. Bioética y bioseguridad en la atención estomatológica al paciente con VIH/sida. Rev Ciencias Médicas [Internet]. 2017 Oct [citado 2018 Abr 05];21(5):13-20. Recuperado a partir de: http://scielo.sld.cu/scielo.php?script=sci_arttext\&pid=S156 1-31942017000500004\&lng=es

17. Bestard Echevarría DM, Placeres Collot M, Hernández Castañeda A, Sánchez Martínez R. Modificación en la información sobre la infección por VIH/SIDA del personal estomatológico en 10 años. Archivo Médico de Camagüey [Internet]. 2014 [citado 2018 Abr 5];13(3):[aprox. 0 p.]. Recuperado a partir http://revistaamc.sld.cu/index.php/amc/article/view/240 3

18. Tamayo M. El Proceso de la Investigación Científica. 4a Ed. México: LIMUSA; 2004.

19. Colombia. Ministerio de Salud. Resolución $\mathrm{N}^{\circ} 008430$ de 1993 Por la cual se establecen las normas científicas, técnicas y administrativas para la investigación en salud. [Internet]. Bogotá. (4 de Octubre de 1993). Recuperado a partir
https://www.minsalud.gov.co/sites/rid/Lists/BibliotecaDig ital/RIDE/DE/DIJ/RESOLUCION-8430-DE-1993.PDF

20. Estrada JH, Ramírez DA. Manifestaciones orales asociadas a la infección por el VIH/sida en la etapa de expansión de la terapéutica antirretro-viral: una década en revisión (20002010). Univ Odontol. [Internet]. 2014 [citado 2018 Abr 06];33(71):19-28. Recuperado a partir de: http://dx.doi.org/10.11144/Javeriana.uo33-71.moai

21. Bedoya G. Revisión de las normas de bioseguridad en la atención odontológica, con un enfoque en VIH/SIDA. Univ. Odontol [Internet]. 2010 [citado el 2015 Oct 20];29(62):4551. Recuperado a partir de: http://www.redalyc.org/articulo.oa?id=231216375006> ISSN 0120-4319

22. Harris-Ricardo J, Díaz-Caballero AJ, Fortich-Mesa N. Oral lesions associated with antiretroviral treatment and viral load in HIV/AIDS patients. Rev Cubana Invest Bioméd [Internet]. 2013 [citado 2018 Abr 05];32(3):284-292. Recuperado a partir de: http://scielo.sld.cu/scielo.php?script=sci_arttext\&pid=S086 4-03002013000300004\&lng=es

23. Real-Cotto JJ, González-Fernández JG, Irrazabal Bohorquez AT, Ramírez Amaya JE, Roby-Arias AJ, Hernández Navarro MI. Conocimiento sobre el VIH/Sida en estudiantes de Odontología de la Universidad de Guayaquil. EDUMECENTRO [Internet]. 2017 Dic [citado $2018 \mathrm{Abr}$ 04];9(4):6-18. Recuperado a partir de: http://scielo.sld.cu/scielo.php?script=sci_arttext\&pid=S207 7-28742017000400002\&lng=es

24. Ramírez V, López V, Anaya G, Lara N. Experiencias de pacientes con VIH/SIDA y respuestas de odontólogos ante el tratamiento dental en la Ciudad de México. Rev. ADM [Internet]. 2008 [citado el 2015 Oct 20];65(3):133-140. Recuperado a partir de: http://www.medigraphic.com/pdfs/adm/od2008/od083e.pdf

25. García C, Sierra J, Vidal Y, Contreras D, Martínez E, Agudelo A. Percepción de personas VIH/SIDA sobre la consulta odontológica. Rev. Cubana. Estomatol [Internet]. 2008;45(1):1-8.[consultado el 2015 Oct 20]. Recuperado a partir de: http://web.archive.org/web/20100416014406/http://bvs. sld.cu/revistas/est/vol45_1_08/est05108.htm

26. César Discacciati JA, Lacerda E. Atendimento odontológico ao portador do HIV: medo, preconceito e ética profissional. Rev. panam. salud pública [Internet]. 2001;9(4):234-239 [consultado el 2015 Oct 20]. Recuperado a partir de: https://scielosp.org/article/rpsp/2001.v9n4/234-239/pt/

27. Dávila M, Mujica M, Eugel E. Experiencia y opinión de las personas que viven con VIH/SIDA sobre la atención odontológica. Rev. odontol. Andes [Internet]. 2010 [citado el 2015 Oct 20];5(1):4-12. Recuperado a partir de: http://erevistas.saber.ula.ve/index.php/odontoula/article/ view/7185/7060

28. Bermúdez-Román V, Bran-Piedrahita L, Palacios-Moya L, Posada-Zapata IC. Influencia del estigma en torno al VIH en el acceso a los servicios de salud. Salud pública Méx [revista en la Internet]. 2015 Jun [citado 2018 Abr 05]; 57(3):252-9. Recuperado a partir de: http://www.scielo.org.mx/scielo.php?script=sci_arttext\&pi $\mathrm{d}=$ S0036-36342015000300014\&lng=es

29. Moral J, Segovia MP. Discriminación en mujeres que viven con VIH/SIDA. Revista Iberoamericana de Psicología y Salud [Internet]. 2011 [citado 2018 Abr 02];2(2):185-206. 
Recuperado a partir de: http://www.redalyc.org/articulo.oa?id=245118507004

30. Tamayo-Zuluaga B, Macías-Gil Y, Cabrera-Orrego R, HenaoPelaéz JN, Cardona-Arias JA. Estigma social en la atención de personas con VIH/sida por estudiantes y profesionales de las áreas de la salud, Medellín, Colombia. Rev Cienc Salud. 2015;13(1):9-23.

dx.doi.org/10.12804/revsalud13.01.2015.01
31. Elizondo JE, Treviño A, Violant D. La odontología y el estigma asociado al VIH. Rev Saúde Pública [Internet]. 2015 [citado 2018 Abr 02];49:79. Recuperado a partir de: https://www.scielo.br/pdf/rsp/v49/es_0034-8910-rspS0034-89102015049005877.pdf 